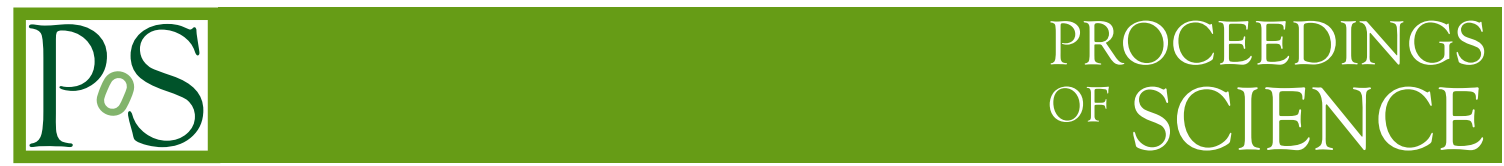

\title{
Background independence of GR and in LQG
}

\section{Jerzy Lewandowski*}

Uniwersytet Warszawski

E-mail: jerzy.lewandowski@fuw.edu.pl

\begin{abstract}
The physical meaning of the diffeomorphisms in the general relativistic theories will be discussed, the issues of time evolution in terms of the Dirac observables and physical Hamiltonian will be addressed. New proposals for geometric deparametrization will be presented. The quantum part of the lecture will concern the canonical LQG. New, improved formulations of the quantum Hamiltonian will be proposed. New applications for LQG will be offered. Original results that will be presented in this lecture were obtained in collaboration with: Dapor, Duch, Kaminski, Swiezewski, Alesci, Assanioussi, Dziendzikowski and Sahlmann.
\end{abstract}

Frontiers of Fundamental Physics 14 - FFP14,

15-18 July 2014

Aix Marseille University (AMU) Saint-Charles Campus, Marseille

\footnotetext{
${ }^{*}$ Speaker.
} 